\title{
Nitrogen gas plasma treatment of bacterial spores induces oxidative stress that damages the genomic DNA
}

\author{
AKIKAZU SAKUDO ${ }^{1,2}$, YOICHI TOYOKAWA ${ }^{1}$, TETSUJI NAKAMURA ${ }^{1}$, \\ YOSHIHITO YAGYU ${ }^{3}$ and YUICHIRO IMANISHI ${ }^{4}$
}

\author{
${ }^{1}$ Laboratory of Biometabolic Chemistry, School of Health Sciences, University of The Ryukyus, Nishihara, \\ Okinawa 903-0215; ${ }^{2}$ Department of Virology, Research Institute for Microbial Diseases, Osaka University, Suita, \\ Osaka 565-0871; ${ }^{3}$ Department of Electrical and Electric Engineering, Sasebo National College of Technology, \\ Nagasaki 857-1193; ${ }^{4}$ NGK Insulators, Mizuho-ku, Nagoya, Aichi 467-8530, Japan
}

Received October 9, 2015; Accepted October 10, 2016

DOI: $10.3892 / \mathrm{mmr} .2016 .5973$

\begin{abstract}
Gas plasma, produced by a short high-voltage pulse generated from a static induction thyristor power supply [1.5 kilo pulse/sec (kpps)], was demonstrated to inactivate Geobacillus stearothermophilus spores (decimal reduction time at $15 \mathrm{~min}, 2.48 \mathrm{~min}$ ). Quantitative polymerase chain reaction and enzyme-linked immunosorbent assays further indicated that nitrogen gas plasma treatment for $15 \mathrm{~min}$ decreased the level of intact genomic DNA and increased the level of 8-hydroxy-2'-deoxyguanosine, a major product of DNA oxidation. Three potential inactivation factors were generated during operation of the gas plasma instrument: Heat, longwave ultraviolet-A and oxidative stress (production of hydrogen peroxide, nitrite and nitrate). Treatment of the spores with hydrogen peroxide $\left(3 \times 2^{-4} \%\right)$ effectively inactivated the bacteria, whereas heat treatment $\left(100^{\circ} \mathrm{C}\right)$, exposure to UV-A $\left(75-142 \mathrm{~mJ} / \mathrm{cm}^{2}\right)$ and $4.92 \mathrm{mM}$ peroxynitrite $\left(\cdot \mathrm{ONOO}^{-}\right)$, which is decomposed into nitrite and nitrate, did not. The results of the present study suggest the gas plasma treatment inactivates bacterial spores primarily by generating hydrogen peroxide, which contributes to the oxidation of the host genomic DNA.
\end{abstract}

\section{Introduction}

Sterilization of medical devices is an important means of reducing the incidence of iatrogenic diseases (1). Recent developments in sterilization methods are aimed at improving the effectiveness, applicability and cost-effectiveness of the process. One such sterilization technique involves treatment with gas plasma, which is generated by removing electrons

Correspondence to: Dr Akikazu Sakudo, Laboratory of Biometabolic Chemistry, School of Health Sciences, University of The Ryukyus, 207 Uehara, Nishihara, Okinawa 903-0215, Japan E-mail: akikazusakudo@gmail.com

Key words: DNA, gas plasma, 8-OHdG, oxidative stress, UV from gases to produce a highly excited mixture of charged nuclei and free electrons. Gas plasma treatment is particularly suitable for the sterilization of thermolabile medical devices (2-4).

Recently, a nitrogen gas plasma instrument (BLP-TES), which generates nitrogen gas plasma using a fast high-voltage pulse from a static induction (SI) thyristor power supply was developed (5-11). Our previous studies (5-11) have demonstrated that nitrogen gas plasma can be utilized to inactivate various microorganisms. However, the mechanisms of action of gas plasma on microorganisms remain to be elucidated. Previous studies have demonstrated that treatment of microorganisms with nitrogen gas plasma may induce changes in their lipids (5), proteins (7) and carbohydrates (8). These findings suggest that the changes induced by sterilizing factors produced during the generation of nitrogen gas plasma may contribute to the inactivation of microorganisms.

Previous studies have demonstrated that nitrogen gas plasma treatment using BLP-TES efficiently inactivates bacteria and viruses as well as endotoxins (5-11). Furthermore, Geobacillus stearothermophilus, which is often used as a biological indicator for gas plasma and is one of the most resistant microbes against physical and chemical treatments, was inactivated by treatment with BLP-TES $(6,12)$. However, it remains unclear how the nitrogen gas plasma generated by BLP-TES inactivates this bacterial spore. In the present study, G. stearothermophilus spores on a stainless steel (SUS) disk and filter paper were treated with nitrogen gas plasma using BLP-TES and any changes to the genomic DNA were analyzed. Furthermore, the effect of various sterilizing factors generated during operation of the BLP-TES instrument were also investigated.

\section{Materials and methods}

Nitrogen gas plasma measurement and treatment. BLP-TES produces nitrogen gas plasma by means of a fast high-voltage pulse applied using a SI thyristor power supply as described in our previous studies (5-7). Cathode electrodes (earth electrodes) were placed between the anode electrodes (high voltage electrodes). All bacterial spore samples and chemical 
indicators were placed on a plastic net on the earth electrodes during nitrogen gas plasma treatment. First, the chamber box containing the sample was decompressed and degassed, and then nitrogen gas (99.9995\%, Okano, Co., Ltd., Kadena, Japan) was introduced. The pressure in the box was maintained at $\sim 0.5$ atmospheres during the discharge at $1.5 \mathrm{kilo}$ pulse/sec (kpps).

Bacterial spores and culture. A filter paper strip and SUS disk coated with G. stearothermophilus ATCC7359 (SGM Biotech Inc., Bozeman, MT, USA) were used for treatment. In certain experiments, a tablet containing G. stearothermophilus EZ-SPORE (Microbiologics, Inc., St. Cloud, MN, USA) was used. Each tablet was suspended in $1 \mathrm{ml}$ of hydration buffer (Microbiologics, Inc.) in accordance with the manufacturer's protocols and then spotted and dried onto a cover glass. The SUS disk, filter paper, or cover glass were treated with nitrogen gas plasma. In all cases, the treated and untreated G. stearothermophilus was mixed with tryptic soy broth (TSB) pH 7.3 \pm 0.2 (Raven Japan Co., Ltd., Koshigaya, Japan) and pH indicator bromocresol purple (BCP) prior to incubation at $56^{\circ} \mathrm{C}$. A color change in the medium as a result of proliferation of the bacteria served as an index of viability.

Polymerase chain reaction (PCR). Plasma-treated or untreated G. stearothermophilus samples on paper filters or SUS disks were resuspended in distilled water and genomic DNA was then eluted by heat treatment at $100^{\circ} \mathrm{C}$ for $15 \mathrm{~min}$. The liberated DNA samples were subjected to PCR amplification comprising 40 cycles of $94^{\circ} \mathrm{C}$ for $1 \mathrm{~min}, 55^{\circ} \mathrm{C}$ for $1 \mathrm{~min}$, and $72^{\circ} \mathrm{C}$ for $1 \mathrm{~min}$, followed by a final incubation at $72^{\circ} \mathrm{C}$ for $10 \mathrm{~min}$. GEOBAC primers (GEOBAC-F and GEOBAC-R) were used in the amplification and based on the $16 \mathrm{~S}-23 \mathrm{~S}$ rRNA gene internal transcribed spacer (ITS) region sequences. GEOBAC-F (5'-TAAGCGTGAGGTCGG TGGTTC-3') targeted the gene of tRNA ${ }^{\text {Ile }}$, and GEOBAC-R (5'-GCGCTCTCGGCTTCTTCCTT-3') targeted the 3' end region of Geobacillus ITS (13). Gstearo-16S primers were targeted to 16S rRNA of G. stearothermophilus and designed to the DNA sequence with Genbank Accession no. EU484358 (www.ncbi.nlm.nih.gov/genbank). The primer sequences were as follows: Forward, 5'-CTTCGGGTCGTAAAGCTCTG-3' termed F1' and reverse, 5'-CCTTTGAGTTTCAGCCTTGC-3' termed R1 and 5'-GAATTCCGCTCTCCTCTCCT-3' termed R2. All the amplifications were performed using a PC320 model thermocycler (Astec Co. Ltd., Kasuya, Japan). The PCR products were initially analyzed by agarose gel electrophoresis and then subsequently verified by DNA sequencing. The PCR products obtained by standard PCR and qPCR were subcloned into pT7Blue T-vector (EMD Millipore, Billerica, MA, USA) and subjected to DNA sequencing (ABI PRISM 3100 Genetic Analyzer; Applied Biosystems; Thermo Fisher Scientific, Inc.) to verify the identity of the amplified product.

Quantitative PCR ( $q P C R)$. The extracted genomic DNA samples were subjected to quantitative PCR using SYBR Premix Ex TaqII (Tli RNase H Plus; Takara Bio Inc., Otsu, Japan) and the following primers for 16S rRNA of G. stearothermophilus (Genbank Accession no. EU484358): Forward, 5'-CACACTGGGACTGAGACACG-3' and reverse,
5'-CATTGCGGATTCCCTAC-3' for region 1; forward, 5'-ACGGTACCTCACGAGAAAGC-3' and reverse, 5'-TCG CCCCCTACGTATTACC-3' for region 2; and forward, 5'-CAT TCGGTTGGGCACTCTA-3' and reverse, 5'-AAGGGGCAT GATGATTTGAC-3' for region 3. The thermocycling conditions used for the quantitative PCR were as follows: $95^{\circ} \mathrm{C}$ for $30 \mathrm{sec}$, followed by 40 cycles of $95^{\circ} \mathrm{C}$ for $5 \mathrm{sec}$ and $60^{\circ} \mathrm{C}$ for $1 \mathrm{~min}$. Relative quantification was performed using the $2^{-\Delta \Delta \mathrm{Cq}}$ method (14).

Scanning electron microscopy (SEM). The G. stearothermophilus-contaminated paper strips were treated with nitrogen gas plasma (1.5 kpps) for 0 and $15 \mathrm{~min}$, and then fixed with $2 \%$ glutaraldehyde/0.1 M phosphate buffer $(\mathrm{pH}$ 7.4) overnight at $4^{\circ} \mathrm{C}$. The cover glasses were subsequently treated with $2 \%$ osmium tetroxide at $4^{\circ} \mathrm{C}$ for $3 \mathrm{~h}$. Samples were dehydrated through a graded ethanol series (50-100\% ethanol) at room temperature. Finally, the samples were subjected to critical point drying and evaporation coating by osmium plasma ions. SEM was performed using a JSM-6320F (JEOL Ltd., Tokyo, Japan) instrument at $5 \mathrm{kV}$ using a magnification of $\mathrm{x} 20,000$.

Temperature measurements. The temperature in the box during operation of the instrument was measured using THERMO LABEL 5E (NiGK Corporation, Kawagoe, Japan), which was placed on the earth electrode. The ambient temperature in the nitrogen gas plasma instrument box was measured using a fiber optic thermometer (FT1420A; Takaoka Electric MFG. Co. Ltd., Tokyo, Japan) during nitrogen gas plasma treatment.

Measurement of ultraviolet $(U V)$ radiation. Indicator label-H (NiGK Corporation) was placed on the earth electrode and treated with nitrogen gas plasma $(1.5 \mathrm{kpps})$ for $0-30 \mathrm{~min}$. Analysis of emission during operation of the nitrogen gas plasma instrument was performed using a USB multichannel spectrophotometer (S-2431; Soma Optics, Ltd., Tokyo, Japan).

Oxidative stress measurements. Chemical indicators were used to estimate the respective concentration of 2-80 mg/l $\mathrm{NO}_{2}^{-}$(nitrite; Kyoritsu Chemical-Check Lab., Corporation, Tokyo, Japan), 10-500 mg/1 $\mathrm{NO}_{3}^{-}$(nitrate; Kyoritsu Chemical-Check Lab. Corporation) and 0.5-25 mg/1 $\mathrm{H}_{2} \mathrm{O}_{2}$ (Merck Ltd. Tokyo, Japan). The following indicators were used: Quantofix Nitrite (Macherey-Nagel, GmbH \& Co. KG, Düren, Germany), Quantofix Nitrite 3000, Quantofix Peroxide 25, Quantofix Peroxide 100, Nitrite, Quantofix Nitrate and Quantofix Active oxygen, all obtained from Macherey-Nagel $\mathrm{GmbH}$, Duren, Germany), which were placed on a plastic net on the earth electrodes prior to treatment with nitrogen gas plasma using a BLP-TES device at $1.5 \mathrm{kpps}$ for 0-30 min.

Treatment with reagents, heating or $U V$. For heat treatment, a suspension of G. stearothermophilus at $3.1 \times 10^{4}$ colony-forming unit $(\mathrm{CFU}) / \mathrm{ml}$ was incubated at temperatures ranging from $40-100^{\circ} \mathrm{C}$ for 30 min using a block incubator (BI-516S; Astec Co. Ltd). For UV treatment, filter papers contaminated with G. stearothermophilus $\left(2.1 \times 10^{6} \mathrm{CFU}\right)$ were exposed to long wavelength UV-A and short wavelength $\mathrm{UV}-\mathrm{C}$ radiation from a handheld UV transilluminator (UVGL-58; UVP, Inc., Upland, 
$6 \mathrm{~h}$

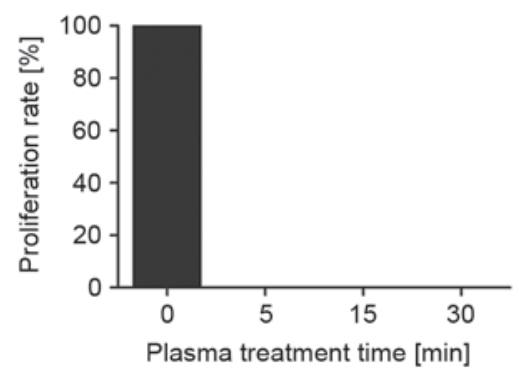

$6 \mathrm{~h}$

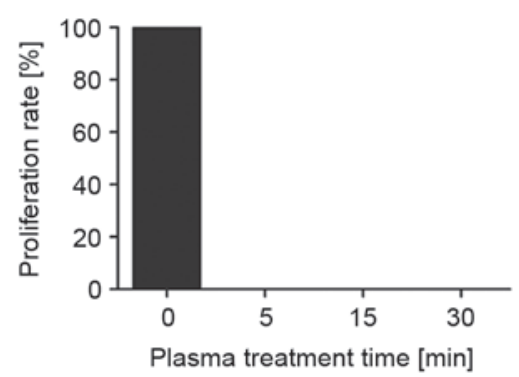

$24 \mathrm{~h}$

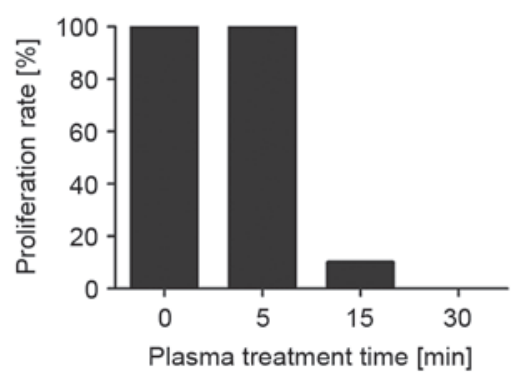

$24 \mathrm{~h}$

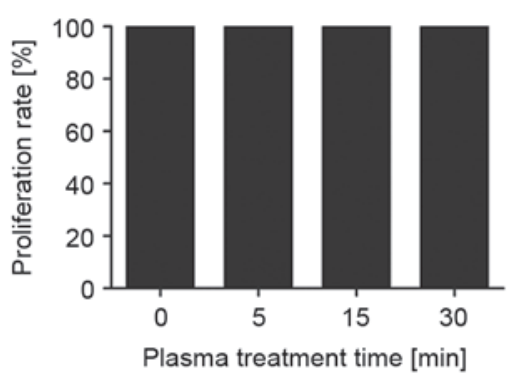

$48 \mathrm{~h}$

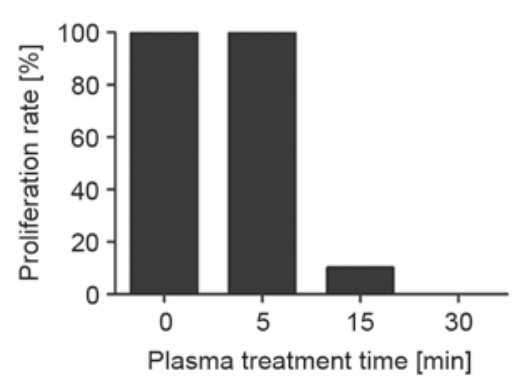

Strip

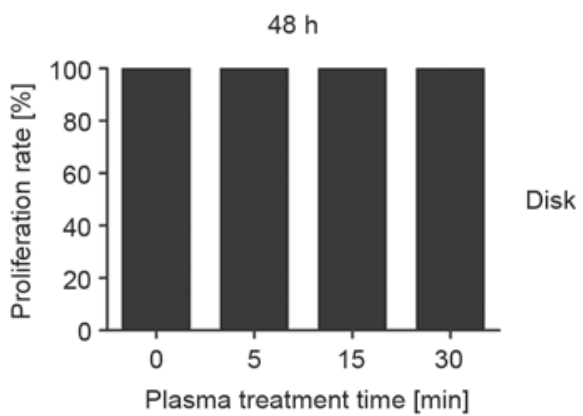

Figure 1. Inactivation of Geobacillus stearothermophilus by nitrogen gas plasma treatment. A total of ten filter papers (strip) and ten SUS disks (disk) contaminated with spores of $G$. stearothermophilus at $1.4 \times 10^{6} \mathrm{CFU}$ and $1.9 \times 10^{6} \mathrm{CFU}$, were treated with nitrogen gas plasma at $1.5 \mathrm{kpps}$ for 0,5 , 15 and 30 min using a BLP-TES device. The BLP-TES instrument generates gas plasma by a short high-voltage pulse generated from a static induction thyristor power supply. The treated bacterial spores were incubated in tryptic soy broth medium, which included the $\mathrm{pH}$ indicator bromocresol purple, at $56^{\circ} \mathrm{C}$ for 6,24 and $48 \mathrm{~h}$. Proliferation of G. stearothermophilus in the medium was monitored by the development of a yellow color due to acidification. The proliferation of G. stearothermophilus following nitrogen gas plasma treatment (1.5 kpps) was then carefully monitored. The growth of the bacteria following a $6 \mathrm{~h}$ incubation was $100 \%$ for the untreated samples and $0 \%$ for samples treated with nitrogen gas plasma (5-30 min treatment period) recovered from filter papers and SUS disks. However, prolonged incubation $(24$ and $48 \mathrm{~h}$ ) of the spores recovered from filter papers gave $100 \%$ growth for the untreated sample and sample treated for $5 \mathrm{~min}, 10 \%$ after $15 \mathrm{~min}$ treatment and $0 \%$ after $30 \mathrm{~min}$ treatment. By contrast, all G. stearothermophilus samples recovered from SUS disks, even those subjected to $30 \mathrm{~min}$ nitrogen gas plasma treatment, proliferated to $100 \%$ after 24 and $48 \mathrm{~h}$ incubation. SUS, stainless steel; CFU, colony-forming units.

CA) for $30 \mathrm{~min}$ on each side of the paper strip (distance from lamp to paper strip, $1.3 \mathrm{~cm})$. The energy $\left(\mathrm{mJ} / \mathrm{cm}^{2}\right)$ of UV-A and UV-C was estimated on the basis of a color change of UV indicator (UV label-H). Samples of G. stearothermophilus suspension $\left(3.1 \times 10^{4} \mathrm{CFU} / \mathrm{ml}\right)$ were subjected to oxidative stress by incubation in the presence of hydrogen peroxide (0-3\%; Wako Pure Chemical Industries, Ltd., Osaka, Japan), peroxynitrite (0-4.92 mM; Dojindo Molecular Technologies, Inc., Kumamoto, Japan) or 3-(4-morpholinyl)sydnonimine hydrochloride(SIN-1; $1.0 \mathrm{mM}-1.0 \times 10^{-5} \mathrm{mM}$; Dojindo Molecular Technologies, Inc.), which produces superoxide anions and nitric oxide resulting in the generation of peroxynitrite.

Analysis of DNA oxidation by enzyme-linked immunosorbent assay (ELISA). G. stearothermophilus samples on filter papers were frozen at $-80^{\circ} \mathrm{C}$ overnight and dipped into $500 \mu 1$ of High Molecular Weight (HMW) buffer containing $10 \mathrm{mM}$ Tris- $\mathrm{HCl}$ ( $\mathrm{pH} 8.0), 0.1 \mathrm{M}$ ethylenediaminetetraacetic acid ( $\mathrm{pH} 8.0$ ), and $0.5 \%$ sodium dodecyl sulfate prior to boiling for $10 \mathrm{~min}$. Following centrifugation at $10,000 \mathrm{x} g$ for $15 \mathrm{~min}$, an aliquot $(50 \mu 1)$ of the supernatant was subjected to the ELISA. To detect 8-hydroxy-2'-deoxyguanosine (8-OHdG), Highly Sensitive 8-OHdG Check ELISA kit (Japan Institute for the Control of Aging, NIKKEN SEIL Co., Ltd., Fukuroi, Japan) and 8-OHdG Assay Preparation reagent set (Wako Pure Chemical Industries, Ltd.) was used in accordance with the manufacturer's protocols. In this kit, a competitive ELISA utilizing monoclonal antibody (clone N45.1), which is highly specific for 8-OHdG, was employed. The concentrations of 8-OHdG was quantified by comparison of absorbance at a wavelength of $450 \mathrm{~nm}$ with standards of $8-\mathrm{OHdG}$ diluted with HMW buffer.

Statistical analysis. The results are presented as the mean \pm standard deviation of replicate experiments $(n=6)$. The statistical analysis of significant difference between plasma-treated and untreated samples was performed by Mann-Whitney $\mathrm{U}$ test. $\mathrm{P}<0.05$ was considered to indicate a statistically significant difference.

\section{Results}

Nitrogen gas plasma treatment decreased the number of viable bacteria spores. The inactivation efficiency of the nitrogen gas plasma was investigated (Fig. 1). The bacterial spores of G. stearothermophilus were spotted and dried onto a SUS disk or filter paper and treated with nitrogen gas plasma at $1.5 \mathrm{kpps}$ for $0,5,15$ or $30 \mathrm{~min}$. The spores were then incubated in TSB medium containing BCP at $56^{\circ} \mathrm{C}$ for 6,24 and $48 \mathrm{~h}$ and the change in $\mathrm{pH}$ of the culture broth was used as the index of bacterial proliferation. The results after $6 \mathrm{~h}$ incubation of the spores taken from the SUS disk (initial population, $1.9 \times 10^{6}$ $\mathrm{CFU}$ ) and filter paper (initial population, $1.4 \times 10^{6} \mathrm{CFU}$ ) indicated that nitrogen gas plasma treatment decreased the number 


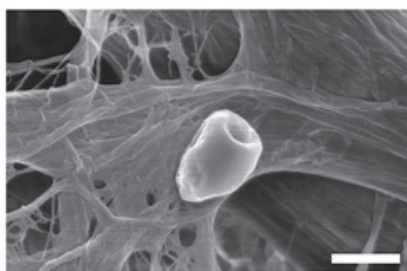

Untreated

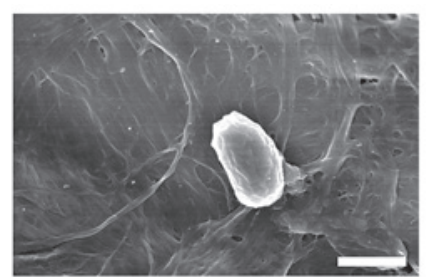

Plasma-treated
Figure 2. Slight changes in surface morphology of G. stearothermophilus following treatment with nitrogen gas plasma. G. stearothermophilus on filter paper was treated with nitrogen gas plasma (1.5 kpps for $15 \mathrm{~min}$ ) using the BLP-TES instrument (plasma-treated). An identical sample was also prepared that was not treated with nitrogen gas plasma (untreated). The surface morphologies of the spores from the two samples were then compared by scanning electron microscopy using a JSM-6320F instrument at $5 \mathrm{kV}$ with a magnification of $x 20,000$. A slight increase in surface roughness was observed following treatment with nitrogen gas plasma. Scale bar indicates $1 \mu \mathrm{m}$.

of viable bacterial spores on the surface of the two materials. However, longer incubation of the samples (24 and $48 \mathrm{~h}$ ) demonstrated that the inactivation efficiency was greater for the spores recovered from the filter paper compared with those from the SUS disk.

The fraction negative method, calculated using the Halvorson-Ziegler formula (15) and Stumbo-Murphy-Cochran procedure (16), indicated the decimal reduction time ( $D$-value) for the filter paper samples was $2.48 \mathrm{~min}$ at $15 \mathrm{~min}$ of treatment, while the $D$ value for the SUS disk could not be calculated, suggesting lower inactivation efficiency in the SUS disk.

No major changes to spores were observed following nitrogen gas plasma treatment. SEM analysis was performed to investigate the effect of nitrogen gas plasma treatment on the structure of the bacterial spore surface (Fig. 2). Careful examination indicated no evidence of shrunken or aggregated spores after nitrogen gas plasma treatment. However, spores subjected to nitrogen gas plasma treatment for 15 min exhibited a slight increase in surface roughness compared with untreated spores.

DNA oxidation was induced by nitrogen gas plasma treatment. Potential changes to the bacterial genomic DNA induced by nitrogen gas plasma treatment were observed (Figs. 3 and 4). SUS disks (disk) and filter papers (strip) were treated with nitrogen gas plasma for 0 and $15 \mathrm{~min}$ and then subjected to PCR analysis using the three pairs of primers designed against the sequence of $G$. stearothermophilus 16S rRNA (F1+R1, F1+R2, and GEOBAC-F+GEOBAC-R; Fig. 3). Agarose gel electrophoresis indicated that no PCR products were amplified from genomic DNA samples of G. stearothermophilus after 15 min treatement with nitrogen gas plasma on a SUS disk or filter paper. By contrast, PCR products of the anticipated size were generated from the untreated samples. Quantitative analysis using qPCR, which employed three pairs of primers designed against three different regions of $16 \mathrm{~S}$ rRNA (region 1-3), demonstrated a decrease of intact genomic DNA in the nitrogen gas plasma treated bacterial spores of G. stearothermophilus compared with untreated samples (Fig. 4). The quantity of intact DNA detected in the nitrogen gas plasma treated samples was $\sim 1 / 10$ that of the untreated

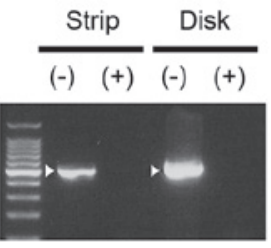

$\mathrm{F} 1+\mathrm{R} 1$

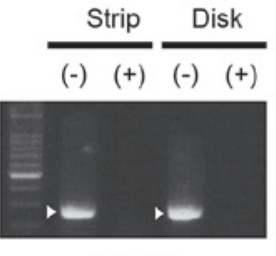

$\mathrm{F} 1+\mathrm{R} 2$

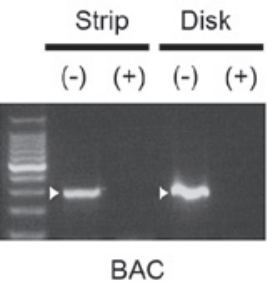

Figure 3. Nitrogen gas plasma treatment of G. stearothermophilus results in damage to the genomic DNA. Filter papers (strip) and stainless steel disks (disk) containing G. stearothermophilus at $2.6 \times 10^{6}$ and $2.1 \times 10^{6} \mathrm{CFU}$, respectively, were either treated with nitrogen gas plasma at $1.5 \mathrm{kpps}$ for 15 min (+) or untreated (-). Genomic DNA was subsequently extracted from the various samples of $G$. stearothermophilus and used as template in PCR with specific sets of primers designed to amplify sections of the 16S rRNA gene (F1+R1, F1+R2 and GEOBAC-F+GEOBAC-R). Damage to the genomic DNA induced by nitrogen gas plasma treatment was analyzed as an index of amplification of intact DNA by PCR (indicated by arrow heads). A DNA size marker (100 bp) was run on the left hand lane of the gel. CFU, colony-forming units; PCR, polymerase chain reaction.

samples. DNA sequence analysis demonstrated that in each case the anticipated product had been amplified. Specifically, 99-87\% (F1+R1), 99-96\% (F1+R2) for Genbank Accession no. EU484358, and 100\% (GEOBAC-F+GEOBAC-R) for Genbank Accession no. EU157949, 99\% (region 1), 100\% (region 2) and $100 \%$ (region 3) for Genbank Accession no. KJ722527. Furthermore, the ELISA demonstrated a significant increase in the level of 8-OHdG in G. stearothermophilus containing filter paper and SUS disk following nitrogen gas plasma treatment ( $\mathrm{P}<0.01$; Fig. 5). 8-OHdG is a product of oxidative damage to DNA formed by hydroxyl radicals, singlet oxygen and direct photodynamic action (17). Thus, the results indicate nitrogen gas plasma treatment of G. stearothermophilus spores induces oxidation of the DNA.

Generation of heat, $U V$-A and oxidative stress in the BLP-TES instrument. Previous studies have confirmed that at least three variables are generated during operation of the nitrogen gas plasma instrument BLP-TES that may be responsible for its sterilizing action, namely heat, UV-A and oxidative stress (10). This is similar to other gas plasma instruments $(18,19)$. Temperature measurement of the sample box in the BLP-TES instrument using a fiber thermometer gave readings of $65^{\circ} \mathrm{C}$ at $7.5 \mathrm{~min}, 75^{\circ} \mathrm{C}$ at $15 \mathrm{~min}$, and $85^{\circ} \mathrm{C}$ at $30 \mathrm{~min}$ during operation of the device. UV measurements using a paper indicator indicated a color change in a time-dependent manner (equivalent to $0 \mathrm{~mJ} / \mathrm{cm}^{2}$ at $0 \mathrm{~min}, 25 \mathrm{~mJ} / \mathrm{cm}^{2}$ at $7.5 \mathrm{~min}$, and $50 \mathrm{~mJ} / \mathrm{cm}^{2}$ at $30 \mathrm{~min}$ ). Consistent with these findings, UV-A was detected by analysis of the emission using a spectrophotometer (S-2431; data not shown). In addition, the levels of chemicals were further estimated by the index of color change. The increase of hydrogen peroxide depended on treatment time and was estimated to be $7.5 \pm 0.9 \mathrm{mg} / \mathrm{l}$ at $30 \mathrm{~min}$. Production of nitrite was also observed during operation of the instrument and was estimated to be $5.5 \pm 0.7 \mathrm{mg} / \mathrm{l}$ at $30 \mathrm{~min}$, while that of nitrate was $92.7 \pm 19.0 \mathrm{mg} / \mathrm{l}$ at $30 \mathrm{~min}$ (data not shown).

The individual contribution of these three variables (heat, UV, and oxidative stress) to inactivation efficiency was examined. Following exposure to each of these variables, at a level equivalent to that observed during operation of the gas 
Table I. Effect of heat treatment for 30 min on the viability of G. stearothermophilus.

Temperature $\left({ }^{\circ} \mathrm{C}\right)$

\begin{tabular}{lllllllllllll}
\cline { 2 - 6 } Viability & 40 & 50 & 60 & 65 & 70 & 75 & 80 & 85 & 90 & 95 \\
\hline Cell growth & + & + & + & + & + & + & + & + & + & +
\end{tabular}

G. stearothermophilus $\left(3.1 \times 10^{4} \mathrm{CFU} / \mathrm{ml}\right)$ was heat treated $\left(40-100^{\circ} \mathrm{C}\right)$ for $30 \mathrm{~min}$, and then incubated in tryptic soy broth medium containing bromcresol purple for $72 \mathrm{~h}$. Bacterial proliferation was determined by monitoring the development of a yellow color in the medium. Plus $(+)$ indicates that proliferation of G. stearothermophilus was observed.

Table II. Effect of UV treatment for $30 \mathrm{~min}$ on the viability of G. stearothermophilus.

\begin{tabular}{lcc}
\hline Viability & UV-A & UV-C \\
\hline Cell growth $(\%)$ & $100(3 / 3)$ & $0(0 / 3)$ \\
\hline
\end{tabular}

Filter papers containing G. stearothermophilus $\left(2.1 \times 10^{6}\right.$ colony forming units) were treated with longwave UV-A and shortwave UV-C using a UV lamp (UVGL-58) for $30 \mathrm{~min}$ at room temperature. Samples were then incubated in tryptic soy broth medium containing bromcresol purple for $72 \mathrm{~h}$, and the development of a yellow color in the medium was monitored. UV-C treatment $(>300$ $\mathrm{mJ} / \mathrm{cm}^{2}$ ) inactivated G. stearothermophilus, whereas UV-A treatment (75-142 mJ/cm²) did not. UV-A, ultraviolet-A; UV-C, ultraviolet-C.

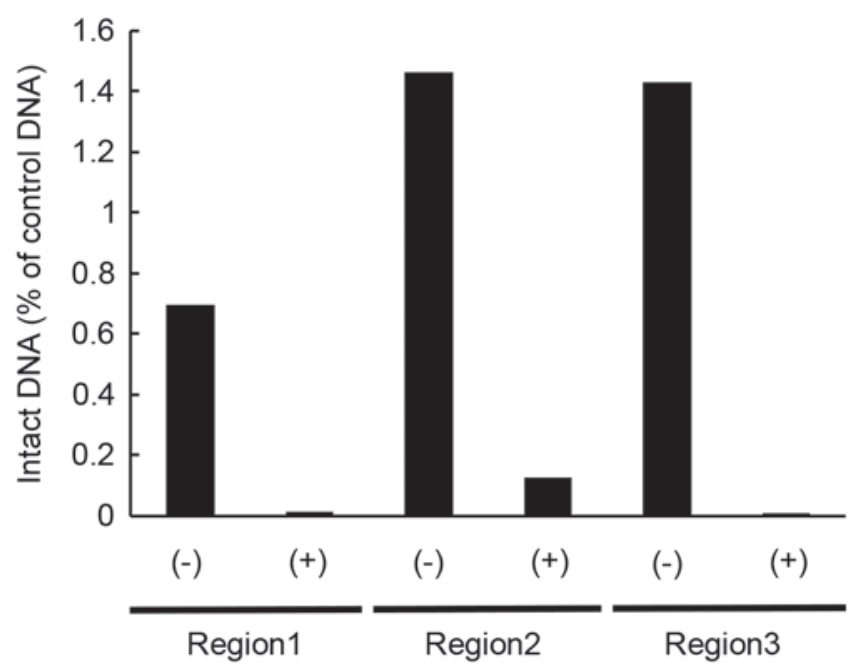

Figure 4. Decrease in quantity of intact genomic DNA of G. stearothermophilus following nitrogen gas plasma treatment. Filter papers containing G. stearothermophilus at $2.1 \times 10^{6}$ colony-forming units were either untreated (-) or treated with nitrogen gas plasma at $1.5 \mathrm{kpps}$ for $15 \mathrm{~min}(+)$. Genomic DNA was subsequently extracted from the G. stearothermophilus samples and subjected to quantitative polymerase chain reaction using primers designed against three regions of the 16S rRNA gene (region 1,2, and 3). The results demonstrate that nitrogen gas plasma treatment decreased the level of intact genomic DNA in all three regions.

plasma device, spores of G. stearothermophilus (3.1x10 4 CFU) were incubated for $48 \mathrm{~h}$. The change in color of the medium was then checked against an index to determine the bacterial proliferation. The results indicated that heating at $40-100^{\circ} \mathrm{C}$

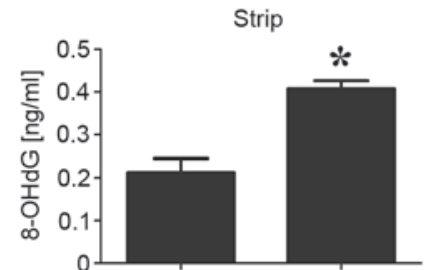

$(-)$
$(+)$

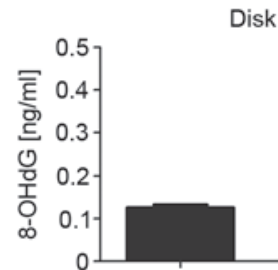

$(-)$
Disk

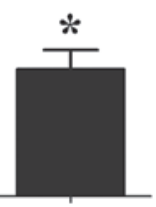

$(+)$
Figure 5. DNA oxidation of G. stearothermophilus by nitrogen gas plasma treatment. Filter papers (strip) and SUS disks (disk) containing G. stearothermophilus at $1.4 \times 10^{6}$ and $1.9 \times 10^{6}$ colony-forming units, respectively, were either untreated (-) or treated with nitrogen gas plasma at $1.5 \mathrm{kpps}$ for 15 min (+). Genomic DNA was subsequently extracted from the various samples of G. stearothermophilus and subjected to an enzyme-linked immunosorbent assay designed to detect 8-OHdG. The levels of 8-OHdG observed in the plasma-treated $(+)$ samples increased compared with the untreated (-) samples of G. stearothermophilus. Statistical analysis was conducted using the Mann-Whitney $\mathrm{U}$ test. ${ }^{*} \mathrm{P}<0.01$ vs the untreated control. 8-OHdG, 8-hydroxy-2'-deoxyguanosine.

for $30 \mathrm{~min}$ did not affect the viability of the bacterial spores (Table I). UV-C treatment for $30 \mathrm{~min}\left(>300 \mathrm{~mJ} / \mathrm{cm}^{2}\right)$ using a transilluminator (UVGL-58) completely inactivated bacterial spores $(100 \%)$, while UV-A treatment $\left(75-142 \mathrm{~mJ} / \mathrm{cm}^{2}\right)$ for the same length of time did not (Table II). In the case of oxidative stress, treatment with peroxynitrite $\left(4.92-4.92 \times 10^{-5} \mathrm{mM}\right)$, SIN-1 $\left(1.0-1.0 \times 10^{-5} \mathrm{mM}\right)$ or hydrogen peroxide $\left(3 \times 2^{-5}-3 \times 2^{-9} \%\right)$ for $30 \mathrm{~min}$ did not inactivate the bacterial spores (Tables III-V). However, exposure of the spores to an elevated level of hydrogen peroxide $\left(3-3 \times 2^{-4} \%\right)$ resulted in their complete inactivation (Table III).

\section{Discussion}

The inactivation rates of bacterial spores on the SUS disks were low compared to those on the filter papers following nitrogen gas plasma treatment at a frequency of $1.5 \mathrm{kpps}$, indicating that different surfaces elicit a different effect on spore viability. These findings are consistent with a previous study, which reported the inactivating efficiency of $G$. stearothermophilus spores on SUS disks was low compared to spores placed on filter papers (12).

In the present study, the $D$ value determined by nitrogen gas plasma treatment of $G$. stearothermophilus on filter papers ( $D$-value $=2.48 \mathrm{~min}$ ) was similar to that of hydrogen peroxide vapor $(2 \mathrm{mg} / 1 ; D$-value=2.1 $\mathrm{min} ; G$. stearothermophilus SUS disk) or steam sterilization $\left(121^{\circ} \mathrm{C} ; D\right.$-value $=2.2 \mathrm{~min}$; G. stearothermophilus filter strip). Thus, the nitrogen gas 
Table III. Effect of hydrogen peroxide treatment for $30 \mathrm{~min}$ on the viability of G. stearothermophilus.

\begin{tabular}{lcccccccccc}
\hline & \multicolumn{10}{c}{$\mathrm{H}_{2} \mathrm{O}_{2}(\%)$} \\
\cline { 2 - 7 } & 3 & $3 \times 2^{-1}$ & $3 \times 2^{-2}$ & $3 \times 2^{-3}$ & $3 \times 2^{-4}$ & $3 \times 2^{-5}$ & $3 \times 2^{-6}$ & $3 \times 2^{-7}$ & $3 \times 2^{-8}$ & $3 \times 2^{-9}$ \\
\hline Cell growth & - & - & - & - & - & + & + & + & + \\
\hline
\end{tabular}

G. stearothermophilus $\left(3.1 \times 10^{4}\right.$ colony forming units $\left./ \mathrm{ml}\right)$ was treated with hydrogen peroxide $\left(3-3 \times 2^{-9} \%\right)$ for 30 min at room temperature. The samples were then incubated in tryptic soy broth medium containing bromcresol purple, for $72 \mathrm{~h}$, and the development of a yellow color in the medium was monitored. Plus (+) indicates that proliferation of G. stearothermophilus was observed, while minus (-) indicates no proliferation.

Table IV. Effect of peroxynitrite $\left(\bullet \mathrm{ONOO}^{-}\right)$treatment for $30 \mathrm{~min}$ on the viability of G. stearothermophilus.

\begin{tabular}{l} 
Viability \\
\cline { 2 - 4 }
\end{tabular}

Table V. Effect of SIN-1 treatment for 30 min on the viabilty of G. stearothermophilus.

\begin{tabular}{l} 
Viability \\
\cline { 2 - 4 }
\end{tabular}

plasma instrument enables efficient inactivation of bacterial spores, equivalent to that observed by exposure to hydrogen peroxide vapor or steam sterilization in the case of samples on filter paper. Hydrogen peroxide vapor is one of the major sterilization methods for thermolabile medical devices (20), while steam sterilization is the conventional sterilization method. However, hydrogen peroxide vapor treatment is not compatible with numerous materials due to its powerful oxidizing activity (21). By contrast, as gas plasma penetration at the surface of the material is slight at $\sim 10-20 \mathrm{~nm}(6)$, the sterilization procedure does not reduce the functional integrity of the medical instrument. However, as certain surface materials, such as SUS, may reduce inactivation efficiency, compatibility of materials should be checked prior to employing nitrogen gas plasma treatment.

One of the sterilization factors is UV-A radiation emitted during the generation of nitrogen gas plasma (10). Thus, it was assumed that gas plasma treatment may elicit its sterilizing action by damaging the genomic DNA of microorganisms. The present study assessed potential damage to the genomic DNA by analyzing the $16 \mathrm{~S}$ rRNA gene of gas plasma treated and untreated spores by PCR and qPCR. The results demonstrated clear evidence that DNA in the bacterial spores is damaged by nitrogen gas plasma treatment. Furthermore, increased levels of 8-OHdG following nitrogen gas plasma suggested that the DNA damage is the result of oxidation. The present study also assessed whether UV-A emitted during the operation of nitrogen gas plasma contributes, at least in part, to the sterilization process. However, UV-A exposure did not efficiently inactivate the bacterial spores. Thus, other factors appear to be predominantly responsible for the microbial inactivation. SEM images demonstrated an irregular surface structure of bacterial spores following treatment with nitrogen gas plasma. The observed changes in cell surface structures are possibly due to oxidative stress produced during gas plasma generation. Therefore, inactivation of bacterial spores during nitrogen gas plasma treatment may the result of changes in the cell surface components, as well as damage to genomic 
DNA as a result of oxidative stress. In summary, the nitrogen gas plasma instrument produces at least three variables, heat, UV-A and oxidative stress, during its operation, which may be responsible for bacterial inactivation. Among these factors, hydrogen peroxide is the most effective for inactivation of G. stearothermophilus. However, there may be a synergistic effect involving the other factors that contribute to the bactericidal activity of nitrogen gas plasma treatment.

Hydrogen peroxide, similarly to formaldehyde or chlorine, exhibits good cell permeability, and may act to reduce the water content of the spores by hydrating dipicolinic acid in the core of the spore (22-24). Thus, hydrogen peroxide generated from the nitrogen gas plasma instrument, in addition to oxidizing the bacterial spore surface, may exert its effect by reducing the water content in the cytoplasm. In addition, other reactive chemical species may be produced during operation of the BLP-TES device. Indeed, the preliminary data indicated a change in the color of the chemical indicator Quantofix Active oxygen which reacts with a broad range of oxidants, including potassium monopersulfate triple salt $\left(\mathrm{KHSO}_{5}, \mathrm{KHSO}_{4}\right.$ and $\mathrm{K}_{2} \mathrm{SO}_{4}$ ) and hydrogen peroxide, bromine $\left(\mathrm{Br}_{2}\right)$, hypochlorite $\left(\mathrm{OCl}^{-}\right)$, peracetic acid $\left(\mathrm{CH}_{3} \mathrm{COOOH}\right)$ and chlorine $\left(\mathrm{Cl}_{2}\right)(25)$. Thus, other reactive chemical species in addition to hydrogen peroxide may contribute to the observed bacterial inactivation and may indicate a synergistic inactivating effect. Thus, further studies are required in order to identify other reactive chemical species that contribute to the bactericidal action of the BLP-TES instrument.

In conclusion, nitrogen gas plasma treatment is an effective means of sterilization and disinfection, suggesting this methodology has practical potential for sterilization of medical devices in the fields of medicine and dentistry, as well as disinfection in the food industry.

\section{Acknowledgements}

The authors of the present study would like to thank Ms. Haruna Ohshiro and Ms. Rika Imamura (Laboratory of Biometabolic Chemistry, School of Health Sciences, University of the Ryukyus, Nishihara, Japan) for their technical assistance and Dr. Naohiro Shimizu (NGK Insulators, Mizuho-ku, Japan) for their technical support. The current study was supported by a Grant-in-Aid for the Promotion of Basic Research Activities for Innovative Biosciences from the Bio-oriented Technology Research Advancement Institution, the Science and Technology Research Promotion Program for Agriculture, Forestry, Fisheries and Food Industry (grant no. 26015A), and a Grant-in-Aid from the Amano Institute of Technology, as well as Grants-in-Aid for Scientific Research on Innovative Areas (grant nos. 22110514, 24110717 and 16K04997) from the Japan Society for the Promotion of Science.

\section{References}

1. McDonnell GE: Antisepsis, Disinfection, and Sterilization: Types, Action, and Resistance. ASM Press, Washington, D.C, 2007.

2. Sakudo A and Shintani H: Sterilization and disinfection by plasma: Sterilization mechanisms, biological and medical applications. Nova Science Publishers, Inc., New York, 2011.
3. Laroussi M: Low temperature plasma-based sterilization: Overview and state-of-the-art. Plasma Process Polym 2: 391-400, 2005.

4. Moisan M, Barbeau J, Crevier MC, Pelletier J, Philip N and Saoudi B: Plasma sterilization: Methods and mechanisms. Pure Appl Chem 74: pp349-358, 2002.

5. Sakudo A, Shimizu N, Imanishi $Y$ and Ikuta $K: N_{2}$ gas plasma inactivates influenza virus by inducing changes in viral surface morphology, protein, and genomic RNA. Biomed Res Int 2013: 694269,2013

6. Shintani H, Shimizu N, Imanishi Y, Sekiya T, Tamazawa K, Taniguchi A and Kido N: Inactivation of microorganisms and endotoxins by low temperature nitrogen gas plasma exposure. Biocontrol Sci 12: 131-143, 2007.

7. Sakudo A, Higa M, Maeda K, Shimizu N, Imanishi Y and Shintani H: Sterilization mechanism of nitrogen gas plasma: Induction of secondary structural change in protein. Microbiol Immunol 57: 536-542, 2013.

8. Sakudo A, Misawa T, Shimizu N and Imanishi Y: $\mathrm{N}_{2}$ gas plasma inactivates influenza virus mediated by oxidative stress. Front Biosci (Elite Ed) 6: 69-79, 2014.

9. Shintani H, Sakudo A, Burke P and McDonnell G: Gas plasma sterilization of microorganisms and mechanisms of action. Exp Ther Med 1: 731-738, 2010.

10. Maeda K, Toyokawa Y, Shimizu N, Imanishi Y and Sakudo A: Inactivation of Salmonella by nitrogen gas plasma generated by a static induction thyristor as a pulsed power supply. Food Control 52: 54-59, 2015.

11. Sakudo A, Toyokawa Y and Imanishi Y: Nitrogen Gas Plasma Generated by a Static Induction Thyristor as a Pulsed Power Supply Inactivates Adenovirus. PLoS One 11: e0157922, 2016.

12. Kawamura K, Sakuma A, Nakamura Y, Oguri T, Sato N and Kido N: Evaluation of bactericidal effects of low-temperature nitrogen gas plasma towards application to short-time sterilization. Microbiol Immunol 56: 431-440, 2012.

13. Kuisiene N, Raugalas J, Stuknyte M and Chitavichius D: Identification of the genus Geobacillus using genus-specific primers, based on the 16S-23S Rrna gene internal transcribed spacer. FEMS Microbiol Lett 277: 165-172, 2007.

14. Livak KJ and Schmittgen TD: Analysis of relative gene expression data using real-time quantitative PCR and the 2(-Delta Delta C(T)) Method. Methods 25: 402-408, 2001.

15. Halvorson HO and Ziegler NR: Application of Statistics to Problems in Bacteriology: I. A Means of Determining Bacterial Population by the Dilution Method. J Bacteriol 25: 101-121, 1933.

16. Stumbo CR, Murphy JR and Cochran J: Nature of Thermal Death Time Curves for P.A. 3679 and Clostridium botulinum [1950]. Food Technol 4: 321-326, 1950.

17. Valavanidis A, Vlachogianni T and Fiotakis C: 8-hydroxy-2'deoxyguanosine (8-OhdG): A critical biomarker of oxidative stress and carcinogenesis. J Environ Sci Health C Environ Carcinog Ecotoxicol Rev 27: 120-139, 2009.

18. Laroussi M and Leipold F: Evaluation of the Roles of Reactive Species, Heat and UV radiation in the Inactivation of bacterial Cells by Air Plasmas at Atmospheric Pressure. Int J Mass Spectrom 233: 81-86, 2004

19. Laroussi M, Mendis DA and Rosenberg M: Plasma Interaction with Microbes. New J Phys 5: 1-41, 2003.

20. Shintani H: Application of vapor phase hydrogen peroxide sterilization to endoscope. Biocontrol Sci 14: 39-45, 2009.

21. Shintani H: Simultaneous Achievement of Sterility Assurance Level (SAL) of $10^{-6}$ and Material and Functional Compatibility in Gas Plasma Sterilization Running Title: Simultaneous SAL and Compatibility. Pharm Regul Aff 4: 131, 2015.

22. Eskenazi S, Bychkowski OE, Smith M and MacMillan JD: Evaluation of glutaraldehyde and hydrogen peroxide for sanitizing packaging materials of medical devices in sterility testing. J Assoc Off Anal Chem 65: 1155-1161, 1982.

23. Kenar L, Ortatatli M, Yaren H, Karayilanoglu T and Aydogan H: Comparative sporicidal effects of disinfectants after release of a biological agent. Mil Med 172: 616-621, 2007.

24. Setlow B, Yu J, Li YQ and Setlow P: Analysis of the germination kinetics of individual Bacillus subtilis spores treated with hydrogen peroxide or sodium hypochlorite. Lett Appl Microbiol 57: 259-265, 2013.

25. MACHEREY-NAGEL GmbH \& Co. KG. QUANTOFIX ${ }^{\circledR}$ Active oxygen. ftp://ftp.mn-net.com/english/Instruction_leaflets/ QUANTOFIX/91349en.pdf\#search=\%27Quantofix+Active+ oxygen\%27. Accessed 25 August 2015. 